\title{
Osthole Improves Cognitive Function of Vascular Dementia Rats: Reducing A $\beta$ Deposition via Inhibition NLRP3 Inflammasome
}

\author{
Yiwei Liu, ${ }^{a, b, c}$ Xia Chen, ${ }^{a, b, c}$ Qihai Gong, ${ }^{a, b, c}$ Jingshan Shi, ${ }^{a, b, c}$ and Fei Li*a, ${ }^{*, b, c}$ \\ ${ }^{a}$ Department of Pharmacology, Key Laboratory of Basic Pharmacology of Ministry of Education and Joint \\ International Research Laboratory of Ethnomedicine of Ministry of Education, Zunyi Medical University; \\ Zunyi 563000, China: ${ }^{b}$ Key Laboratory of Basic Pharmacology of Guizhou Province, Zunyi Medical University; \\ Zunyi 563000, China: and ${ }^{c}$ School of Pharmacy, Zunyi Medical University; Zunyi 563000, China. \\ Received February 5, 2020; accepted June 18, 2020
}

Vascular dementia (VD) is a common neurodegenerative disease, and the cognitive dysfunction is a major manifestation of VD. Lots of evidences showed that beta-amyloid (A $\beta$ ) deposition and neuroinflammation act as vital elements in the progress of VD. The previous studies showed that osthole (OST) can improve the cognitive function of VD and Alzheimer's disease (AD). However, the effect of OST on A $\beta$ in VD brain is still unclear. Chronic cerebral hypoperfusion $(\mathrm{CCH})$ of rats were used to investigate the effect of OST on A $\beta$ through nod-like receptor protein 3 (NLRP3) inflammasome in this study. Morris Water Maze and Y-maze were used to test the spatial learning, memory and working abilities. Hematoxylin-eosin (H\&E) and Nissl staining were used to observe the morphology and number of hippocampal neurons. Immunofluorescence staining was used to observe the number of microglia activated. Western blot was used to detect the expression of proteins. The study results showed that OST obviously enhanced the spatial learning, memory and working abilities induced by modified bilateral common carotid artery occlusion (BCCAO) in rats, improved the pathological damage of hippocampal neurons induced by BCCAO in rats, inhibited the activation of microglia induced by BCCAO in rats. Furthermore, this study also discovered that OST reduced A $\beta$ deposition in VD hippocampus via inhibition the NLRP3 inflammasome. Together, these results suggest that OST reduces A $\beta$ deposition via inhibition NLRP3 inflammasome in microglial in VD.

Key words osthole; vascular dementia; chronic cerebral hypoperfusion; beta-amyloid; inflammasome

\section{INTRODUCTION}

Cognitive dysfunction is a major manifestation of vascular dementia (VD) which is a neurodegenerative disease. ${ }^{1)}$ Among all dementia patients, Alzheimer's disease (AD) ranked first, and VD second. VD currently has no effective treatment, as a result, the family and society have suffered a great economic burden, it is very urgent to develop a new drug or therapy for it. $^{2)}$ VD has a variety of different subtypes which exists the heterogeneity among them, but the study indicated that one of the starting conditions for VD is chronic cerebral hypoperfusion $(\mathrm{CCH})$, after angiogenesis and collateral circulation are established, $\mathrm{CCH}$ status will be reversed. ${ }^{3)} \mathrm{CCH}$ is a chronic and long-term state in which cerebral blood flow continues to decrease, thereby impairing the function of neurons which leads to cognitive dysfunction. ${ }^{4)}$ In order to imitate VD model, according to literatures the $\mathrm{CCH}$ rats in this study were operated the modified bilateral common carotid artery occlusion (BCCAO). ${ }^{5)}$

Currently, VD has quite complex pathogenesis, such as beta-amyloid $(\mathrm{A} \beta)$ deposition, tau hyperphosphorylation, inflammatory response etc. ${ }^{6-8)}$ Furthermore, lots of evidences indicated that the rats induced by BCCAO emerged progressive cognitive dysfunction, $\mathrm{A} \beta$ deposition and increasing of inflammatory response, the process was reversed after treatment with medication. ${ }^{9,10)}$ Neuroinflammation has both positive and negative sides, either too strong or too weak inflammation is harmful to neurons. ${ }^{11,12)}$ The study showed that amyloid precursor protein (APP) can maintain the plasticity of neurons, conserve neurons, and then alleviate the cognitive dysfunction, a large amount of APP appears in hippocampus of rats induced by BCCAO, and APP is decomposed into $\mathrm{A} \beta$ by $\beta$-site APP cleavage enzyme (BACE1). ${ }^{13)}$ A large number of $\mathrm{A} \beta$ aggregates to form $\mathrm{A} \beta$ deposition which will lead to microglial activation, and then over-expression of nod-like receptor protein 3 (NLRP3) inflammasome in microglial to scavenge $\mathrm{A} \beta$ and cause neuronal inflammatory apoptosis, and neuronal inflammatory apoptosis in turn result in over-expression of APP eventually causes the inflammatory reaction of vicious circle. ${ }^{14)}$ In the Pharmacopoeia of the People's Republic of China, there is a traditional Chinese medicine called Shechuangzi from the fruit of Cnidium monnieri (L.), and it is used to cure skin diseases and gynecological diseases, Shechuangzi has been proved to possess multiple pharmacological characteristics, including cardiovascular activity, effects on the respiratory system, neuroprotective activity, anti-anxiety and memory improvement effects, effects on the reproductive system, regulatory effects on the endocrine system, antiinflammatory and anti-pruritic effects, immunomodulatory effect, anti-cancer effects, liver protective effect, anti-osteoporotic activity, other effects etc., so far, many different chemical components have been separated from Shechuangzi, including coumarins, volatile oils, chromones, triterpenoids, glycosides, glucides, other compounds etc. ${ }^{15}$ )

Osthole (OST) is a coumarin compound from Chinese herb Shechuangzi. ${ }^{16)}$ After oral administration of OST, it can be detected in the brain tissue of mouse at $5 \mathrm{~min}$ and $10 \mathrm{~h}$ later, and a certain concentration of OST can be maintained for a long time in the brain tissue of mouse, indicating that OST is absorbed rapidly and eliminated slowly in the brain tissue. ${ }^{17}$ 
It has been reported that OST has a wide range of pharmacological activities, for example, anti-tumor, anti-seizure antihypertension etc. ${ }^{18)}$ Numerous interesting researches indicated that OST exists neuroprotection in different animal models, for instance, improving cognitive function of $\mathrm{AD}$ model, preventing cerebral ischemia-reperfusion injury, enhancing adult neurogenesis by up-regulating brain derived neurotrophic factor (BDNF). ${ }^{19-21)}$ But it is not still clear that the effect of OST on $\mathrm{A} \beta$ deposition and on neuroinflammation in $\mathrm{CCH}$ animal model. Therefore, this study aims to investigate whether OST exerts therapeutic effect on VD, and explores the possible underlying mechanisms.

\section{MATERIALS AND METHODS}

Drugs OST $\left(\mathrm{C}_{15} \mathrm{H}_{16} \mathrm{O}_{3}, \quad \mathrm{MW}\right.$ : 244.29, 7-methoxy-8-isopentenoxycoumarin, purity $\geq 99.90 \%$ by LC-MS) was purchased from MedChemExpress.cn (Shanghai, China) (CAS: 484-12-8), and it is mainly through chemical synthesis, tween 80 as a solvent to dissolve it.

Animals Sprague-Dawley (SD, adult, male) rats $(260 \pm 20 \mathrm{~g})$ were purchased from the Changsha Tianqin Biotechnology Corporation Ltd. (Changsha, China; SPF grade, Certificate No. SCXK2014-0011). The temperature $\left(22 \pm 1{ }^{\circ} \mathrm{C}\right)$ and humidity $(55 \pm 10 \%)$ are constant in the SPF-grade room, and it turns on at 8:00 and off at 20:00 every day. Per cage had five rats which were free to drink water and eat foods. The rats were raised for 2 weeks before experiment, and the animal experiments were followed the Experimental Animal Ethics Committee of Zunyi Medical University.

Model Prepare The rats were subjected to the modified BCCAO which followed anesthesia with $3 \mathrm{~mL} / \mathrm{kg}$ intraperitoneal injections of $2 \%$ sodium pentobarbital. In short, the rats were supine fixed on the rat board, and made a $1 \mathrm{~cm}$ long incision about $0.3 \mathrm{~cm}$ to the left of the center of the neck. The left common carotid artery, vagal nerve, carotid sheath and cervical sympathetic were clearly visible between the sternohyoid and sternomastoid muscles. The two glass needle dividers were used to separate out the left common carotid artery which was tightly ligated with 4-0 silk sutures. Finally, the left incision was sutured with 2-0 silk sutures. After a week, the rats were subjected to the same operation on the right of the neck. The sham-operated rats were subjected the same surgery but not occlusion.

Drug Administration and Experimental Design The rats were randomly assigned to five groups: sham group $(n=12)$, BCCAO group $(n=12), \mathrm{BCCAO}+$ OST 5 group $(n=12), \mathrm{BCCAO}+\mathrm{OST} 10$ group $(n=12)$, and BCCAO + OST 20 group $(n=12)$. The treatment would be started on the 2 th day after the BCCAO surgery, the rats in OST treated groups were received daily oral administration of different doses of OST 5, 10, and $20 \mathrm{mg} / \mathrm{kg}$, respectively for 62 continuous days. Double distilled water of the same volume was given to the sham and BCCAO control groups. On the 63th day after modeling, all rats were sacrificed to prepare for subsequent experiments.

Morris Water Maze Test The Morris Water Maze was used to test the abilities of spatial learning and memory. The task was carried out during days 53-59 after surgery. The Morris Water Maze complete set of equipment mainly consisted of a circular black pool (diameter: 160, height: $50 \mathrm{~cm}$ ), cam- era system and animal behavior trajectory analysis system. There was a platform in the pool, and the water $\left(24 \pm 2^{\circ} \mathrm{C}\right)$ was added to the submerged platform for $1 \mathrm{~cm}$. We divided the pool into four quadrants: the first quadrant, the second quadrant, the third quadrant and the fourth quadrant. The platform was at the midpoint of the first quadrant. The rats were put into the water with their heads facing the pool wall, and the order was randomly selected one of the other three quadrants except the first quadrant every day. The time of finding the underwater platform was recorded as the escape latency. If the rats failed to find the platform in $120 \mathrm{~s}$, the escape latency was recorded as $120 \mathrm{~s}$, and then the rats were guided to the platform to rest for $15 \mathrm{~s}$. The experiment lasted for $7 \mathrm{~d}$, there were 3 trials per animal every day, and the TopScan-Topview Behavior Analyzing System (TopScan Version 3.00) recorded the escape latency.

Y-maze Test The Y-maze tested the ability of spatial working memory. There were $\mathrm{A}, \mathrm{B}$, and $\mathrm{C}$ three identical arms at $120^{\circ}$ in the Y-maze (length: 50, width: 10, height: $20 \mathrm{~cm}$ ). We gently lowered the rat's head toward the end of an arm, keeping the whole process quiet and letting it move freely in the Y-maze for $10 \mathrm{~min}$. A successful spontaneous reaction alternation behavior is that the head and limbs of the rat completely enter the arm, and they must successively enter and exit the three different arms. After a test, we sprayed the device with $10 \%$ alcohol, scrubbed the device evenly with an absorbent cloth, and finally dried the device with dry toilet paper in the experiment. " $N$ " represented the total number of arm entries, and " $n$ " represented the sequence of entries. The spontaneous alternation rate $(\%)=[n /(N-2) \times 100]$. The TopScan-Topview Behavior Analyzing System (TopScan Version 3.00) recorded " $N$ " and " $n$."

Hematoxylin-Eosin (H\&E) Staining, Nissl Staining and Immunofluorescence Staining After the Y-maze test, four rats in each group were anesthesia with $3 \mathrm{~mL} / \mathrm{kg}$ intraperitoneal injections of $2 \%$ sodium pentobarbital. $0.1 \mathrm{M}$ phosphate-buffered saline (PBS) and 4\% paraformaldehyde solution were prepared in this study and stored in $4^{\circ} \mathrm{C}$. Four rats were perfused transcardially with $200 \mathrm{~mL}$ PBS and paraformaldehyde solution respectively each group, and then the brain was immediately separated on ice plate. The brain was fixed in the $4 \%$ paraformaldehyde solution for $48 \mathrm{~h}$. Finally, it went through a series of processes: dehydration, paraffin embedding and sectioning for H\&E staining, Nissl staining and Immunofluorescence staining (IBA1, 1:200, Abacm, ab178847, U.K.; IBA1, 1:200, Abacm, ab15690; NLRP3, $1: 200$, Abacm, ab214185). The upright metallurgical microscope (BX43 + DP2b, Olympus, Japan) would be observed the damage of neurons in $\mathrm{CA} 3$ region of hippocampus, the positive fluorescent microscope (MBX53, Olympus) would be observed the number of microglia activated in CA3 region of hippocampus.

Western Blot After the Y-maze test, the rest rats in each group were sacrificed after anesthetized with $3 \mathrm{~mL} / \mathrm{kg}$ intraperitoneal injections of $2 \%$ sodium pentobarbital, and the brains were collected on ice plate to prepare hippocampal tissue homogenate. Main steps: the extraction and quantification of proteins, electrophoresis, electro-transformation, blocking with $5 \%$ non-fat milk, incubation with primary antibodies with rotation at $4^{\circ} \mathrm{C}$, and then incubated with horseradish peroxidase (HRP)-conjugated secondary antibodies, finally 
reacted with enhanced chemiluminescence (ECL). The primary antibodies used in this study included the following: APP (1:1000, Abacm, ab32136), BACE1 (1:1000, Abacm, ab108394), $\mathrm{A} \beta_{1-40}(1: 2000$, Abnova, MAB2675, U.S.A.), A $\beta_{1-42}(1: 1000$, Abcam, ab201060), NLRP3 (1:500, Abacm, ab214185), $\beta$-actin (1:2000, Proteintech, 66009-1-lg, China).<smiles>COc1ccc2ccc(=O)oc2c1CC=C(C)C</smiles>

The secondary antibodies used in this study included the following: Goat Anti-Mouse immunoglobulin G $(\mathrm{IgG})(\mathrm{H}+\mathrm{L})$ (1:5000, Proteintech, SA00001-1) and Goat Anti-Rabbit IgG $(\mathrm{H}+\mathrm{L})(1: 5000$, Proteintech, SA00001-2).

Statistical Analysis SPS 18.0 Software was used for statistical analysis of this study, mean \pm standard error of the mean (S.E.M.) as the expression of data. The experimental results of this study were analyzed by a one-way ANOVA and Bonferroni multiple comparisons test, except the escape latency which was analyzed by repeated measures ANOVA and Bonferroni multiple comparison test. $p<0.05$ was considered statistically significant.

Fig. 1. The Chemical Structure of OST

A

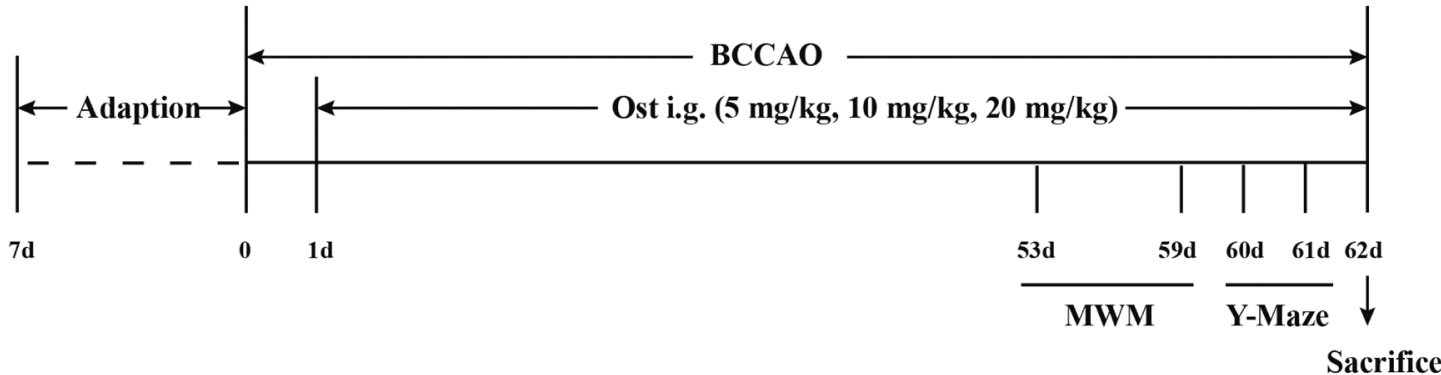

B

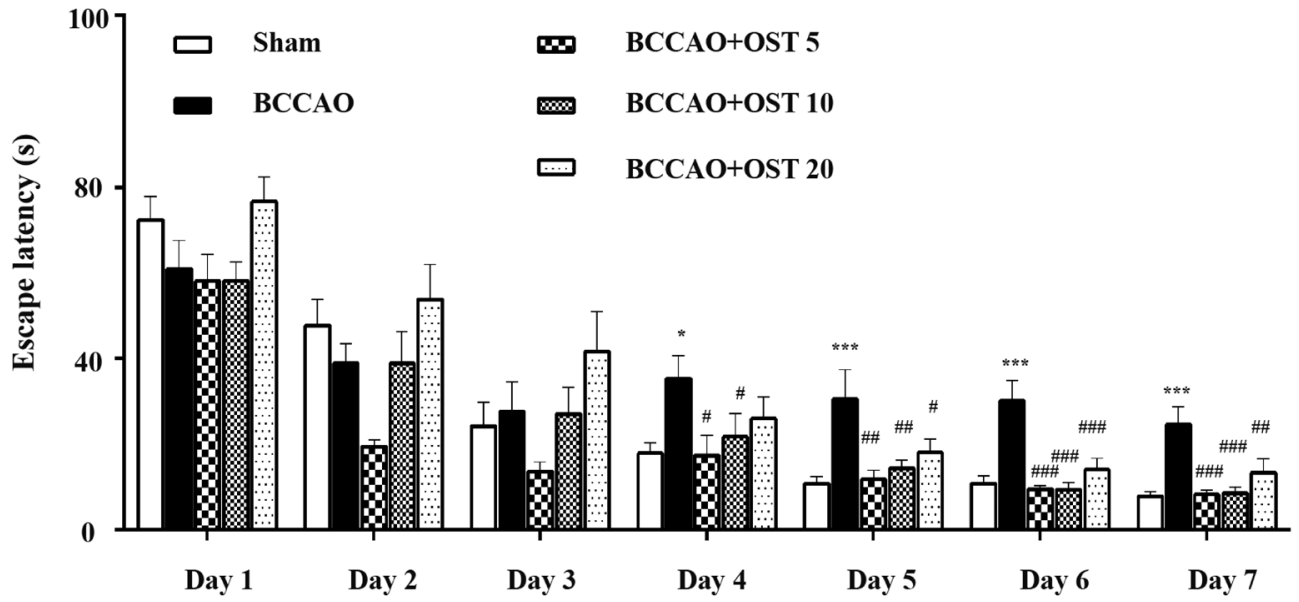

C

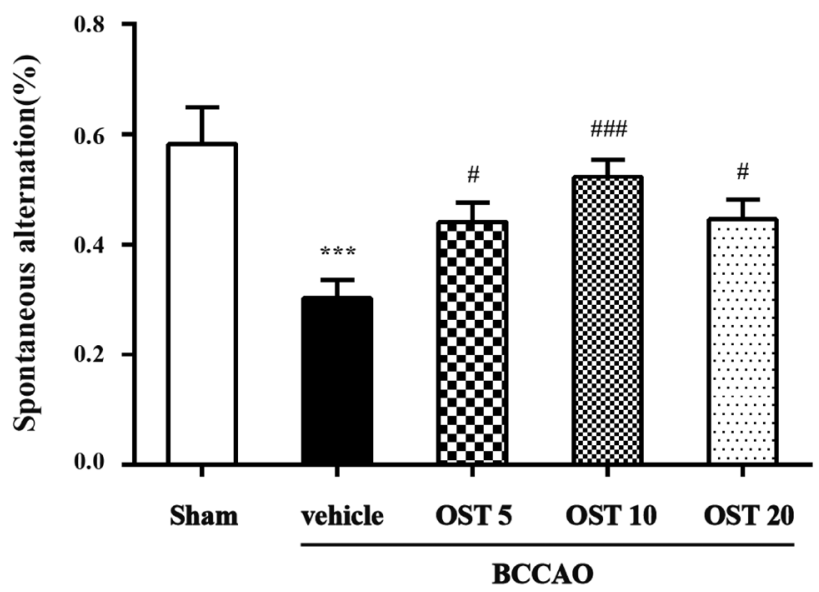

Fig. 2. Effect of OST on Spatial Learning, Memory and Working Deficit of VD Rat

(A) Experimental design: After BCCAO surgery in rats, oral administration of OST 5, 10, and $20 \mathrm{mg} / \mathrm{kg}$ to rats for $61 \mathrm{~d}$. Morris Water Maze test was carried out from day 53 to day 59 after surgery for 1 week. From day 60 to day 61 after surgery, Y-maze test was performed for 2 d. (B) From day 1 to day 7 , the results of the escape latency of rats in each group. (C) The average spontaneous alternation rate of rats in each group. Data were expressed as mean \pm S.E.M. $(n=12) . * p<0.05, * * * p<0.001$ vs. sham, ${ }^{\#} p<0.05,{ }^{\#} p<0.01,{ }^{\# \#} p<0.001$ vs. BCCAO. 


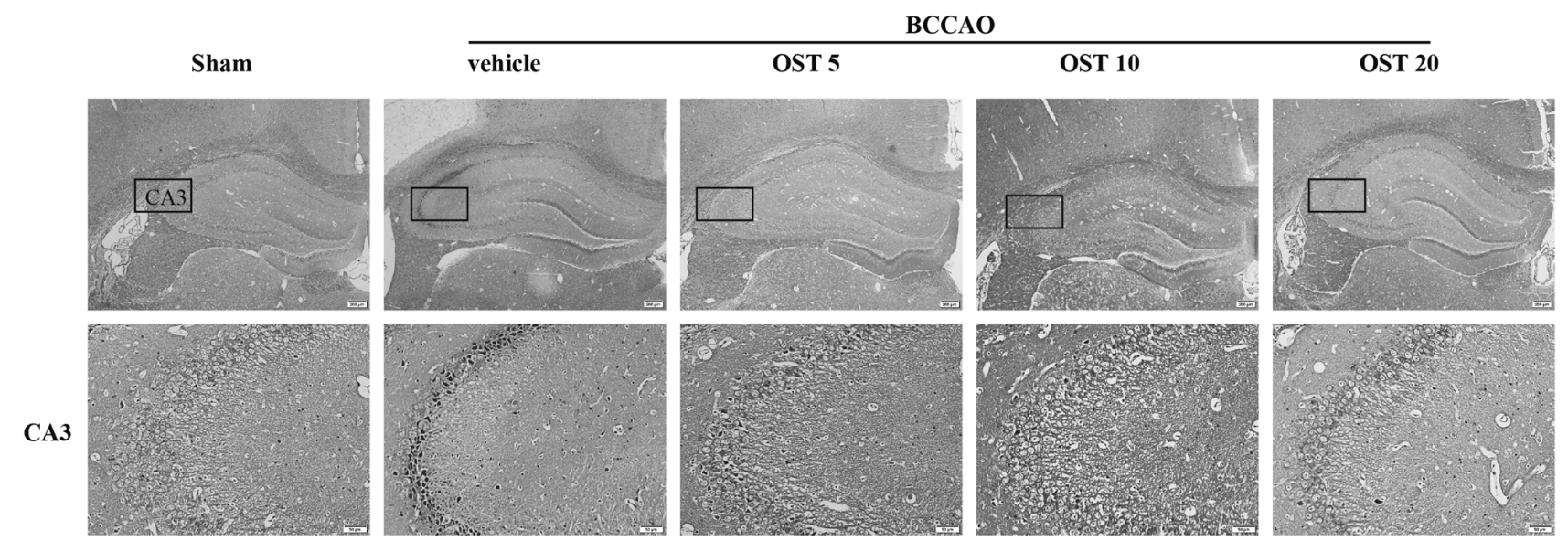

Fig. 3. Effect of OST on the Pathological Damage of Neurons in the Hippocampus of VD Rats

The pathological damage of neurons was examined by H\&E staining (magnification $40 \times$ or $200 \times$, scale bar $=200$ or $50 \mu \mathrm{m}$ ).

A

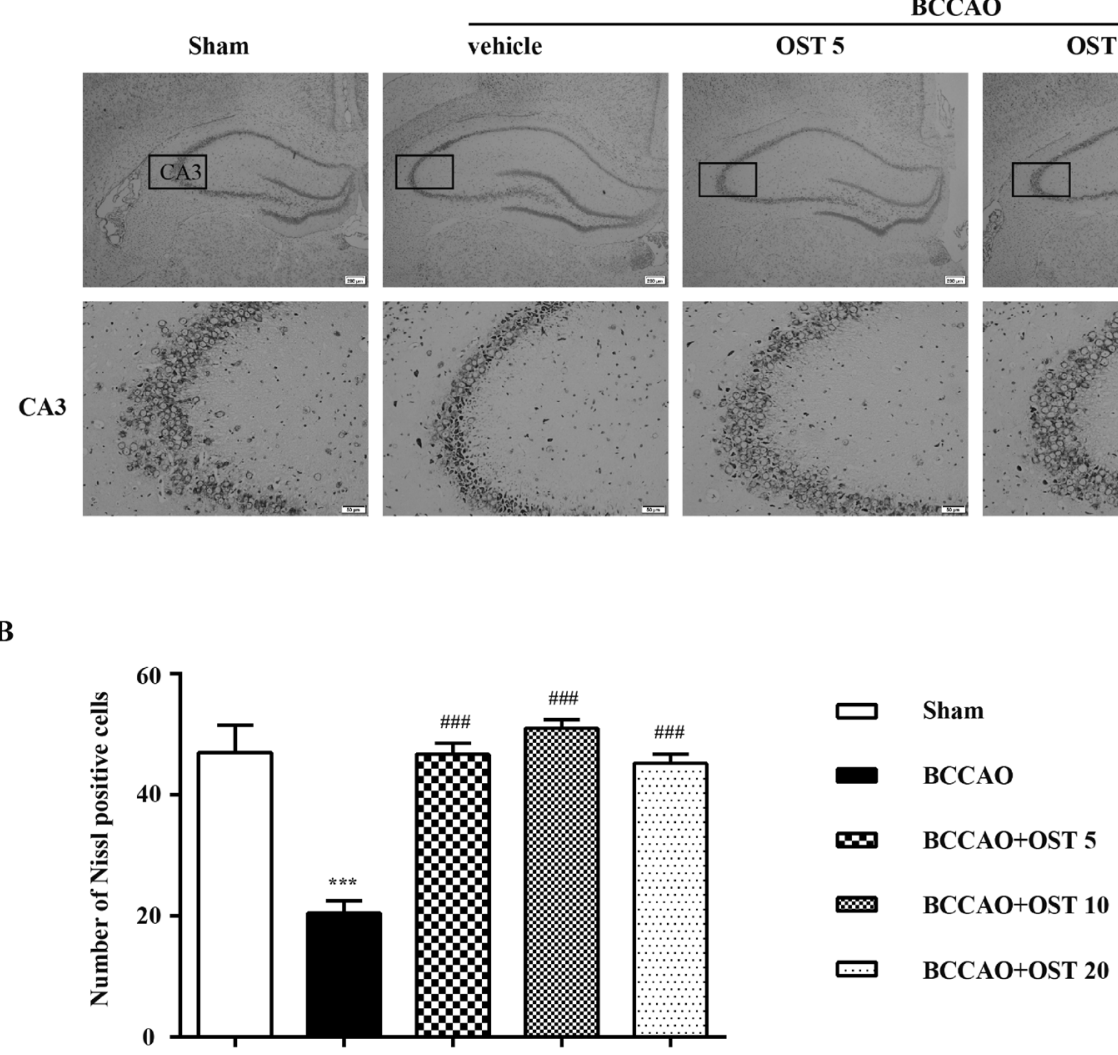

Fig. 4. Effect of OST on the Number of Nissl Bodies and Intact Cells in the Hippocampus of VD Rats

The number of Nissl bodies and intact cells were examined by Nissl staining (magnification $40 \times$ or $200 \times$, scale bar $=200$ or $50 \mu$ m). (A) The typical representative pictures of hippocampal CA3 region. (B) The statistical pictures of hippocampal CA3 region. Data were expressed as mean \pm S.E.M. $(n=4)$. $* * * p<0.001 v s$. sham, $\# \#<0.001$ vs. BCCAO.

\section{RESULTS}

OST Attenuated Spatial Learning, Memory and Working Deficits in VD Rats To investigate whether OST could have an effect on the behavior function, Morris Water Maze test and Y maze test were used in this study. From the day 53 after the BCCAO surgery, the spatial learning and memory abilities were observed by Morris Water Maze test lasted for 1 week (Fig. 2A). The escape latency of each group of rats was significantly shortened followed training. From day 4 to day 7 , the escape latency of the BCCAO model rats was sig- nificantly longer than that of the sham rats (day 4: $p<0.05$, day 5: $p<0.001$, day 6: $p<0.001$, day 7: $p<0.001$ ), however, rats treated with OST in $5,10 \mathrm{mg} / \mathrm{kg}$ and $20 \mathrm{mg} / \mathrm{kg}$ groups significantly shortened the escape latency than BCCAO rats $(p<0.05, p<0.01$ and $p<0.001$ in Fig. 2B).

The spontaneous alternation behavior was used to evaluate the spatial working memory of the rats in the Y-maze test. From the day 60 after surgery, we performed the 2-d Y-maze test (Fig. 2A) to investigate whether OST could attenuate spatial working memory deficit which was induced by BCCAO. The results showed that the spontaneous alter- 
A
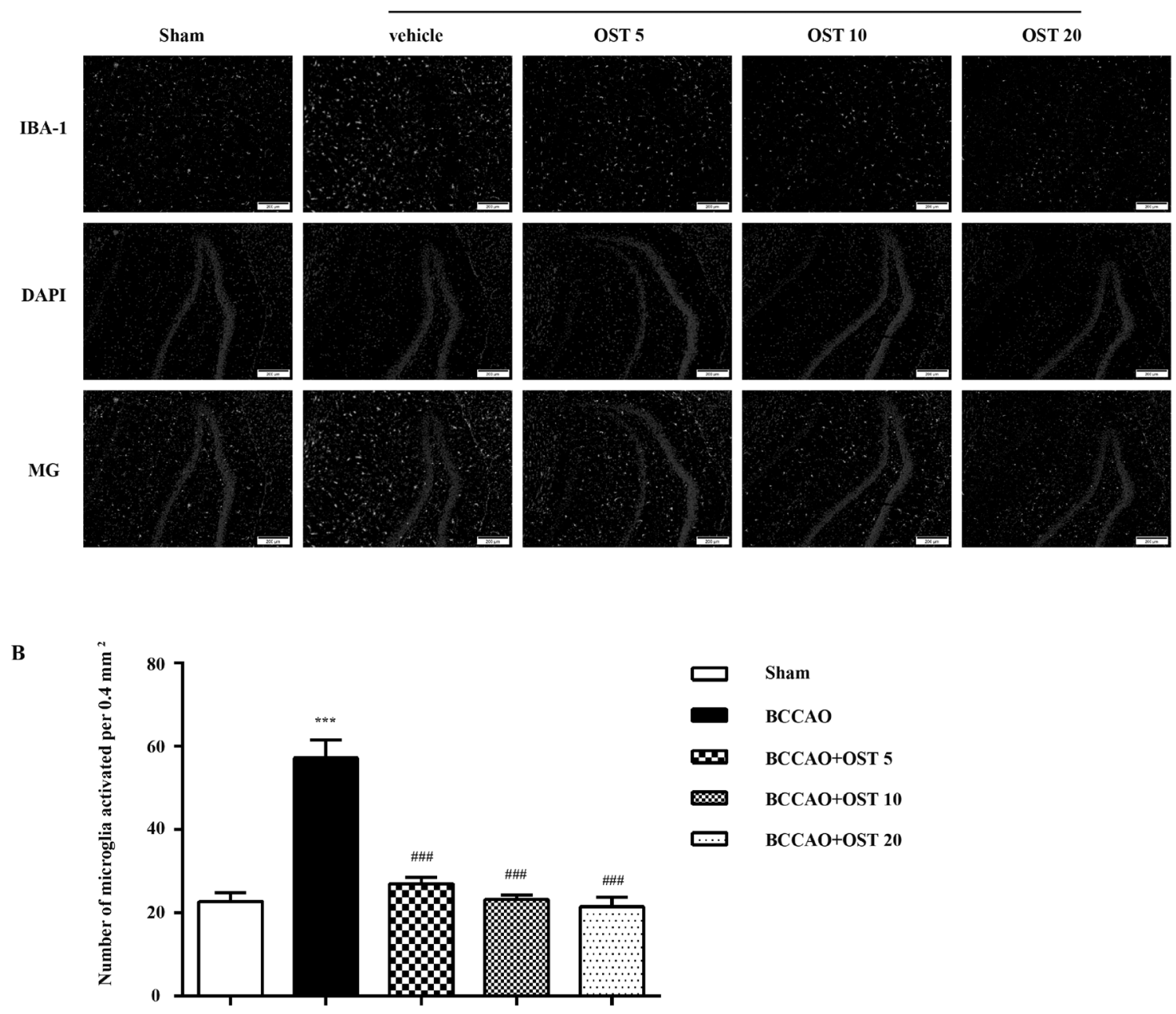

Fig. 5. OST Inhibited the Activation of Microglia in the Hippocampus of VD Rats

The number of microglia activated were examined by immunofluorescence staining with anti-IBA1 antibody (magnification $100 \times$, scale bar $=200 \mu$ m). (A) The typical representative pictures of hippocampal CA3 region. (B) The statistical pictures of hippocampal CA3 region. Data were expressed as mean \pm S.E.M. $(n=4)$. $* * * p<0.001$ vs. sham, ${ }^{\#} p<0.001$ vs. BCCAO.

nation rate of $\mathrm{BCCAO}$ rats was signally lower than that of the sham group $(p<0.001)$, however, rats treated with OST in $5 \mathrm{mg} / \mathrm{kg}(p<0.05), 10 \mathrm{mg} / \mathrm{kg} \quad p<0.001)$, and $20 \mathrm{mg} / \mathrm{kg}$ $(p<0.05)$ groups were observably increased the spontaneous alternation rate compared with BCCAO rats (Fig. 2C).

OST Alleviated the Damage of Neurons in the Hippocampus of VD Rats $H \& E$ and Nissl staining were used to examine the damage of neurons in CA3 region of hippocampus induced by BCCAO. Compared with the sham group, the neurons were obviously pathological damage in CA3 region of $\mathrm{BCCAO}$ rats by $\mathrm{H} \& \mathrm{E}$ stained, however, the neurons damage was distinctly alleviated in rats treated with OST $(5,10$, and $20 \mathrm{mg} / \mathrm{kg}$, respectively) (Fig. 3).

Compared with the sham group, few Nissl bodies and intact cells were found in hippocampal CA3 region of BCCAO rats by Nissl stained $(p<0.001)$, followed OST treatment $(5 \mathrm{mg} / \mathrm{kg}$ $(p<0.001), 10 \mathrm{mg} / \mathrm{kg}(p<0.001), 20 \mathrm{mg} / \mathrm{kg}(p<0.001))$, the number of Nissl bodies and intact cells were visibly increased (Fig. 4). The results of H\&E and Nissl staining showed that OST alleviated the damage of neurons in the hippocampus of VD rats.

OST Inhibited the Activation of Microglia in the Hippocampus of VD Rats Immunofluorescence staining was used to observe the number of microglia activated in CA3 region of hippocampus induced by BCCAO. Compared with the sham group, the number of microglia activated were obviously increased in hippocampal CA3 region of BCCAO rats $(p<0.001)$, followed OST treatment $(5 \mathrm{mg} / \mathrm{kg} \quad(p<0.001)$, $10 \mathrm{mg} / \mathrm{kg}(p<0.001), 20 \mathrm{mg} / \mathrm{kg}(p<0.001))$, the number of microglia activated was visibly reduced. The result of Immunofluorescence staining indicated that OST inhibited the activation of microglia in the hippocampus of VD rats (Fig. 5).

OST Inhibited Microglial Activation and NLRP3 Protein Expression in the Hippocampus of VD Rats In order to explore the relationship of activated microglial activation and NLRP3 in the hippocampus of VD rats, the number of microglial activation and NLRP3 protein expression were examined by double immunofluorescence staining with antiIBA1 and anti-NLRP3 antibodies. Compared with the sham group, the number of microglial activation and NLRP3 protein expression were obviously increased in the hippocampus of VD rats (IBA1: $p<0.001$; NLRP3: $p<0.001$ ), and treatment with OST [5 mg/kg (IBA1: $p<0.001$, NLRP3: $p<0.001$ ), $10 \mathrm{mg} / \mathrm{kg}$ (IBA1: $p<0.001, \quad$ NLRP3: $p<0.001$ ), $20 \mathrm{mg} / \mathrm{kg}$ (IBA1: $p<0.001$, NLRP3: $p<0.001$ ), respectively] evidently reduced the number of microglial activation and NLRP3 pro- 
A

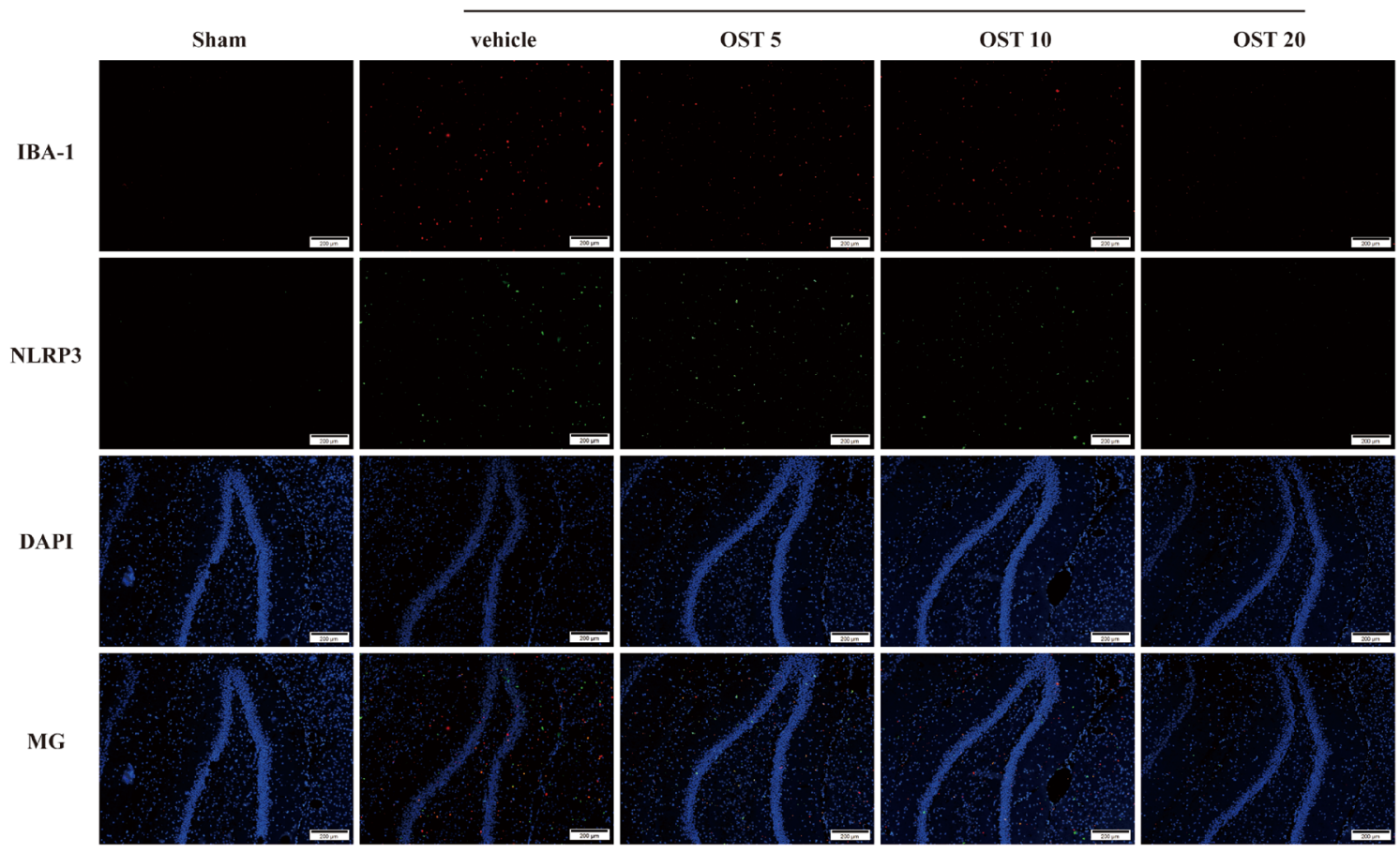

B

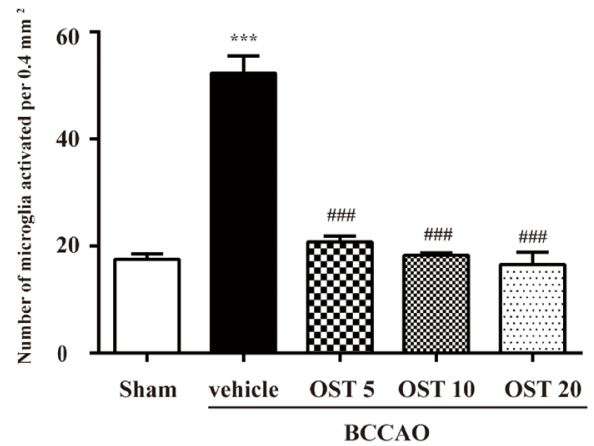

C

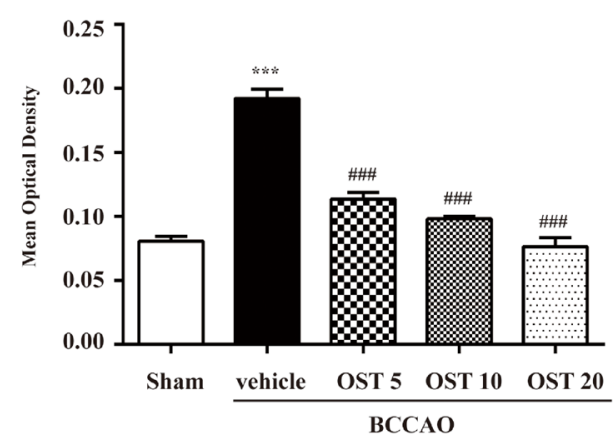

Fig. 6. OST Inhibited Microglial Activation and NLRP3 Protein Expression in the Hippocampus of VD Rats

The number of microglial activation and NLRP3 protein expression were examined by double immunofluorescence staining with anti-IBA1 and anti-NLRP3 antibodies (red fluorescence represented microglia and green fluorescence represented NLRP3 inflammasome, magnification $100 \times$, scale bar $=200 \mu \mathrm{m})$. (A) The typical representative pictures of hippocampal region. (B, C) Quantification of the fluorescence intensity of the staining to assess IBA1 and NLRP3 level. Data were expressed as mean \pm S.E.M. $(n=4) .{ }^{* * *} p<0.001$ vs. sham, ${ }^{\# \#} p<0.001$ vs. BCCAO. (Color figure can be accesed in the online version.)

tein expression compared with BCCAO rats (Fig. 6).

OST Down-Regulated the Level of NLRP3 in the Hippocampus of VD Rats Compared with the sham group, the expression of NLRP3 of hippocampus was obviously increased in the BCCAO model group by Western blot test $(p<0.001)$, and treatment with OST $[5 \mathrm{mg} / \mathrm{kg} \quad(p<0.001), 10 \mathrm{mg} / \mathrm{kg}$ $(p<0.001), 20 \mathrm{mg} / \mathrm{kg}(p<0.001)$, respectively] decreased the level of NLRP3 expression compared with the BCCAO model group (Fig. 7). These findings showed that OST could attenuate NLRP3 expression in the hippocampus of VD rats.

OST Reduced the Levels of $A \beta_{1-40}$ and $A \beta_{1-42}$ Oligomers Deposited in the Hippocampus of VD Rats Compared with the sham group, the deposition of $\mathrm{A} \beta_{1-40}$ and $\mathrm{A} \beta_{1-42}$ oligomers in hippocampus of BCCAO rats were obviously increased in Western blot $\left(\mathrm{A} \beta_{1-40}: p<0.01, \mathrm{~A} \beta_{1-42}: p<0.05\right)$, and treatment with OST $\left[5 \mathrm{mg} / \mathrm{kg}\left(\mathrm{A} \beta_{1-40}: p<0.05, \mathrm{~A} \beta_{1-42}: p<0.01\right)\right.$, $10 \mathrm{mg} / \mathrm{kg} \quad\left(\mathrm{A} \beta_{1-40}: p<0.001, \quad \mathrm{~A} \beta_{1-42}: \quad p<0.01\right), \quad 20 \mathrm{mg} / \mathrm{kg}$
(A $\left.\beta_{1-40}: p<0.001, \mathrm{~A} \beta_{1-42}: p<0.01\right)$, respectively] markedly decreased the levels of $\mathrm{A} \beta_{1-40}$ and $\mathrm{A} \beta_{1-42}$ oligomers (Fig. 8). These results indicated that OST could decrease the deposition of $\mathrm{A} \beta_{1-40}$ and $\mathrm{A} \beta_{1-42}$ oligomers in the VD rat's hippocampus.

OST Decreased the Levels of APP and BACE1 in the Hippocampus of VD Rats In order to study the mechanism of OST inhibiting the increase of $\mathrm{A} \beta$, Western blot detected the protein expression levels of APP and BACE1. Compared with the sham rats, the expression of APP and BACE1 in hippocampus were overtly increased in BCCAO rats (APP: $p<0.001$; BACE1: $p<0.001)$, and treatment with OST [5 mg/kg (APP: $p<0.01$, BACE1: $p<0.01$ ), $10 \mathrm{mg} / \mathrm{kg}$ (APP: $p<0.001$, BACE1: $p<0.001$ ), $20 \mathrm{mg} / \mathrm{kg}$ (APP: $p<0.001$, BACE1: $p<0.001$ ), respectively] distinctly reduced the levels of APP and BACE1 expression compared with BCCAO rats (Fig. 9). 
A

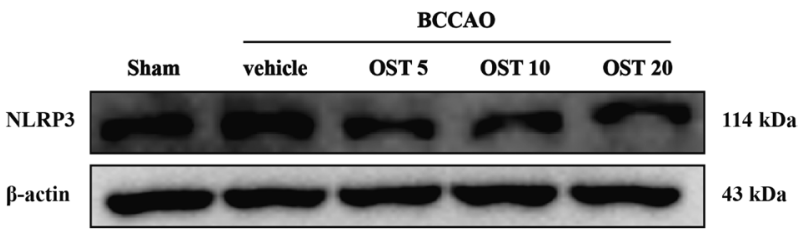

B

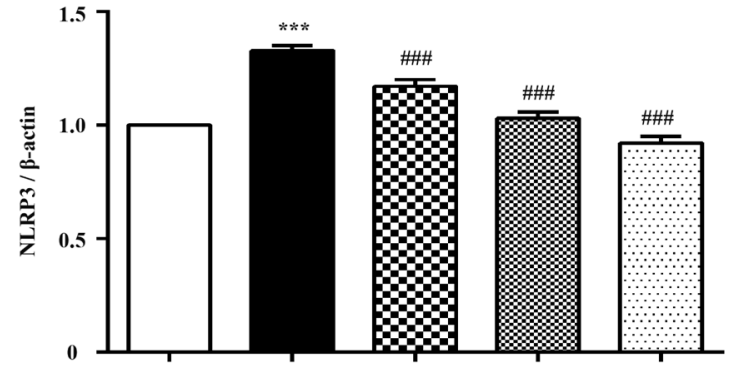

Fig. 7. Effect of OST on Inflammasome NLRP3 in the Hippocampus of VD Rats

Western blot detected the protein level of NLRP3. (A) The antibody-reactive band of the NLRP3. (B) Quantitative analysis of NLRP3. $\beta$-Actin was used to normalize the relative optical density. Data were expressed as mean \pm S.E.M. $(n=5)$. $* * * p<0.001$ vs. sham, \#\# $p<0.001$ vs. BCCAO.

A

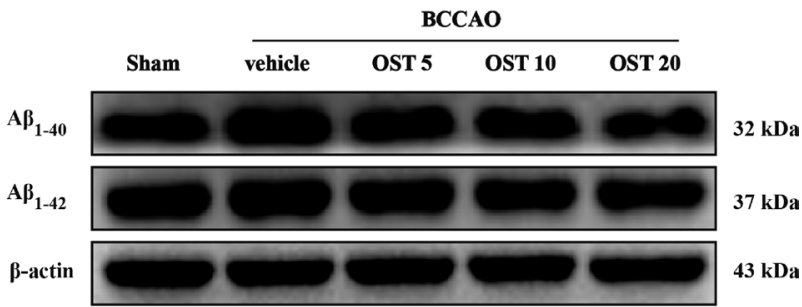

B

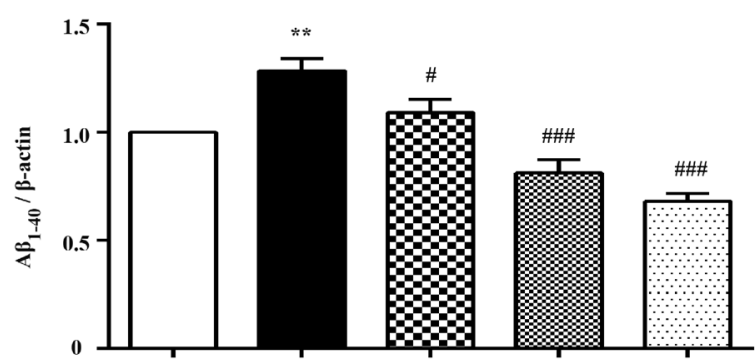

C

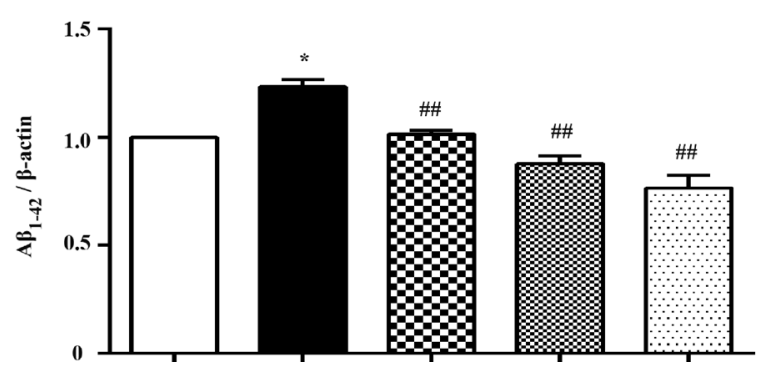

Fig. 8. Effect of OST on the Deposition of $\mathrm{A} \beta_{1-40}$ and $\mathrm{A} \beta_{1-42}$ Oligomers in Hippocampus

Western blot examined the content of $\mathrm{A} \beta_{1-40}$ and $\mathrm{A} \beta_{1-42}$ oligomers of hippocampus tissue. (A) The antibody-reactive bands of the $\mathrm{A} \beta_{1-40}$ and $\mathrm{A} \beta_{1-42}$ oligomers. (B) Quantitative analysis of $\mathrm{A} \beta_{1-40}$ oligomer. (C) Quantitative analysis of $\mathrm{A} \beta_{1-42}$ oligomer. $\beta$-Actin was used to normalize the relative optical density. Data were expressed as mean \pm S.E.M. $(n=5)$. ${ }^{*} p<0.05, * * p<0.01$ vs. sham, ${ }^{*} p<0.05$, ${ }^{\# \#} p<0.01,{ }^{\# \#} p<0.001$ vs. BCCAO.

\section{DISCUSSION}

This study verified that OST attenuated cognitive dysfunction in VD rats induced by BCCAO, evidenced by attenuating spatial learning, memory and working deficits, alleviating the neurons damage and reducing the number of microglia activated in hippocampal CA3 region, decreasing the deposition of $\mathrm{A} \beta_{1-40}, \mathrm{~A} \beta_{1-42}$ oligomers in hippocampus, and downregulating the expressions of APP, BACE1, NLRP3 proteins in the VD rats' hippocampus. Together with these results in the study indicated that the effect of OST on VD rats is related to the regulation of APP-A $\beta$ pathway and inhibition inflammasome in the hippocampus.

The previous studies discovered that rats exhibited a remarkable decrease of cognitive function induced by BCCAO ${ }^{22)}$ The results from Y-maze and Morris Water Maze demonstrated that the prominent spatial learning, memory and working deficits appeared in BCCAO rats of this study. Nevertheless, OST remarkably reversed the cognitive dysfunction after chronic treatment. Moreover, H\&E and Nissl staining disclosed that the neurons damage of hippocampal CA3 area was partially reversed by OST treatment, which was accordance to the behavioral results. This study strongly proved that OST possesses the therapeutic effect on BCCAO inducedcognitive dysfunction.

APP, glycosylated receptor proteins located on the surface

A

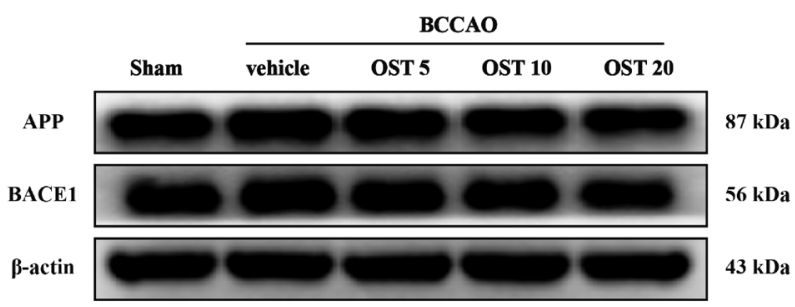

B

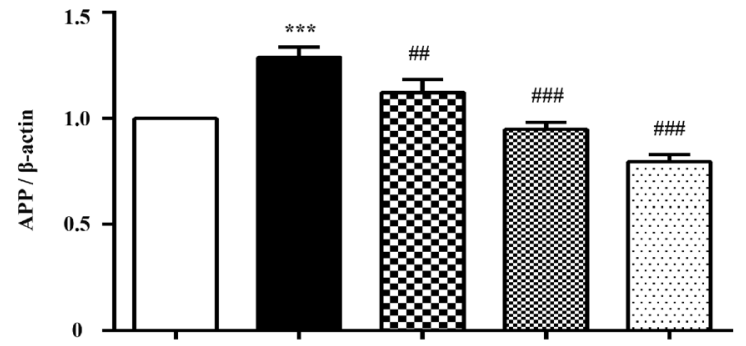

C

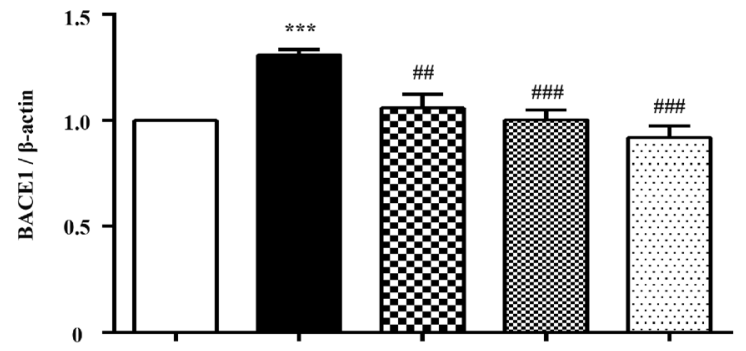

Fig. 9. Effect of OST on APP and BACE1 Proteins Expression in the Hippocampus of VD Rats

Western blot detected the protein expression of APP and BACE1 of hippocampus tissue. (A) The antibody-reactive bands of the APP and BACE1. (B) Quantitative analysis of APP level. (C) Quantitative analysis of BACE1 level. $\beta$-Actin was used to normalize the relative optical density. Data were expressed as mean \pm S.E.M. $(n=5) .{ }^{* * *} p<0.001$ vs. sham, ${ }^{\#} p<0.01,{ }^{\# \#} p<0.001$ vs. BCCAO. 
of cell membranes, is made up of 695 amino acid fragments, as a crucial precursor protein. It can maintain the plasticity of neurons and conserve neurons, it has two decomposition pathways: amyloidogenic pathway and non-amyloidogenic pathway. ${ }^{23)}$ During the early processing of APP, BACE1 is a membrane-bound aspartic protease which is a vital enzyme. $^{24)}$ In the amyloidogenic pathway, APP is decomposed into $\mathrm{SAPP} \beta$ and $\mathrm{C} 99$ by BACE1 at $\mathrm{N}$-terminal of $\mathrm{A} \beta$ area, and C99 is further decomposed by $\gamma$-secretase to produce a large amount of $\mathrm{A} \beta_{1-40}$ and a small amount of $\mathrm{A} \beta_{1-42}$ with neurotoxicity. ${ }^{25)}$ On the other hand, in the non-amyloidogenic, APP is decomposed into neuroprotective soluble amyloid precursor protein $\alpha$ and $\mathrm{C} 83$ by $\alpha$-secretase at $\mathrm{N}$-terminal of $\mathrm{A} \beta$ area, and $\mathrm{C} 83$ is further decomposed into P3 by $\gamma$-secretase, which avoids the generation of $\mathrm{A} \beta .^{26)} \mathrm{A} \beta$ peptide has a $\beta$-lamella secondary structure of polypeptide, and it is made up of 39-43 amino acid fragments. ${ }^{27)} \mathrm{A} \beta$ is normal product in the brain, the amount of physical $\mathrm{A} \beta$ can have the inhibition effect of the over-activation neurons, and it is greatly necessary to keep neurons functioning properly and continuously. $\mathrm{A} \beta_{1-40}$ and $\mathrm{A} \beta_{1-42}$ are major components of amyloid plaques, and its' oligomers exhibited the strongest neurotoxicity. ${ }^{28)}$ Numerous evidences indicated that the imbalance between $\mathrm{A} \beta$ production and clearance in the brain leads to neurodegenerative diseases, such as $\mathrm{AD}$ and $\mathrm{VD}{ }^{29)}$ There are many reasons for the increasing $\mathrm{A} \beta$, it is reported that the neuroinflammation plays a predominant role in $\mathrm{A} \beta$ imbalance. ${ }^{30)}$ In this study, rats induced modified BCCAO showed that APP, BACE1, A $\beta_{1-40}$, $\mathrm{A} \beta_{1-42}$ protein were increased, and microglial activation accompanied with the increase of NLRP3 protein were due to inflammation, however the increase of $\mathrm{A} \beta$ would promote inflammatory response in turn ultimately resulted in a vicious infinite circle.

In conclusion, this study elucidated that OST could obviously enhance learning and memory function and the ability of spatial working memory, improve the pathological damage CA3 area of hippocampus. Furthermore, this study suggested that OST could reduce $\mathrm{A} \beta$ deposition via inhibition overexpression of NLRP3 in microglial to produce anti-neuroinflammatory response in VD.

Acknowledgments The study was supported by National Natural Science Foundation of China (Grant No. 81560594), National key R \& D plan for Research on Modernization of Traditional Chinese Medicine (Grant No. 2017YFC1702005), Post subsidy project of State key R \& D plan in social development field (Grant No. SQ2017YFC170204-05), Program for Changjiang Scholars and Innovative Research Team in University, China (Grant No. IRT_17R113), The "hundred" level of high-level innovative talents in Guizhou Province (Grant No. QKHRCPT 20165684), Guizhou Science and Technology Department (Grant No. QKH-JC-[2016]1137).

Conflict of Interest The authors declare no conflict of interest.

\section{REFERENCES}

1) Zong WJ, Zeng XC, Chen SY, Chen LL, Zhou LP, Wang XT, Gao Q, Zeng GR, Hu K, Ouyang DS. Ginsenoside compound $\mathrm{K}$ attenuates cognitive deficits in vascular dementia rats by reducing the $\mathrm{A} \beta$ deposition. J. Pharmacol. Sci., 139, 223-230 (2019).

2) Yin CX, Deng YY, Liu YG, Gao JM, Yan LL, Gong QH. Icariside II ameliorates cognitive impairments induced by chronic cerebral hypoperfusion by inhibiting the amyloidogenic pathway: involvement of BDNF/TrkB/CREB signaling and up-regulation of PPAR $\alpha$ and PPAR $\gamma$ in rats. Front. Pharmacol., 9, 1211 (2018).

3) Jiang P, Chen L, Sun J, Li JS, Xu J, Liu WY, Feng F, Qu W. Chotosan ameliorates cognitive impairment and hippocampus neuronal loss in experimental vascular dementia via activating the Nrf2-mediated antioxidant pathway. J. Pharmacol. Sci., 139, 105-111 (2019).

4) Li WX, Wei D, Lin JX, Liang JY, Xie XM, Song KP, Huang LA. Dl-3- $n$-Butylphthalide reduces cognitive impairment induced by chronic cerebral hypoperfusion through GDNF/GFR $\alpha 1 /$ Ret signaling preventing hippocampal neuron apoptosis. Front. Cell. Neurosci., 13, 351 (2019).

5) Cechetti F, Worm PV, Pereira LO, Siqueira IR, A Netto C. The modified 2VO ischemia protocol causes cognitive impairment similar to that induced by the standard method, but with a better survival rate. Braz. J. Med. Biol. Res., 43, 1178-1183 (2010).

6) Liu B, Gao JM, Li F, Gong QH, Shi JS. Gastrodin attenuates bilateral common carotid artery occlusion-induced cognitive deficits via regulating $\mathrm{A} \beta$-related proteins and reducing autophagy and apoptosis in rats. Front. Pharmacol., 9, 405 (2018).

7) Chen J, Zhou SN, Zhang YM, Feng YL, Wang S. Glycosides of cistanche improve learning and memory in the rat model of vascular dementia. Eur. Rev. Med. Pharmacol. Sci., 19, 1234-1240 (2015).

8) Sohn E, Kim YJ, Lim HS, Kim BY, Jeong SJ. HwangryunhaedokTang exerts neuropreventive effect on memory impairment by reducing cholinergic system dysfunction and inflammatory response in a vascular dementia rat model. Molecules, 24, 343 (2019).

9) Won JS, Kim J, Annamalai B, Shunmugavel A, Singh I, Singh AK. Protective role of $S$-nitrosoglutathione (GSNO) against cognitive impairment in rat model of chronic cerebral hypoperfusion. J. Alzheimers Dis., 34, 621-635 (2013).

10) Shin JW, Kweon KJ, Kim DK, Kim P, Jeon TD, Maeng S, Sohn NW. Scutellarin ameliorates learning and memory deficit via suppressing $\beta$-amyloid formation and microglial activation in rats with chronic cerebral hypoperfusion. Am. J. Chin. Med., 46, 1203-1223 (2018).

11) Chen $\mathrm{C}$, Wei YZ, He XM, Li DD, Wang GQ, Li JJ, Zhang F. Naringenin produces neuroprotection against LPS-induced dopamine neurotoxicity via the inhibition of microglial NLRP3 inflammasome activation. Front. Immunol., 10, 936 (2019).

12) Zhou JY, Deng YY, Li F, Yin CX, Shi JS, Gong QH. Icariside II attenuates lipopolysaccharide-induced neuroinflammation through inhibiting TLR4-MyD88 NF-kappaB pathway in rats. Biomed. Pharmacother., 111, 315-324 (2019).

13) Rice HC, de Malmazet D, Schreurs A, Frere S, Molle IV, Volkov AN, Creemers E, Vertkin I, Nys J, Ranaivoson FM, Comoletti D, Savas JN, Remaut H, Balschun D, Wierda KD, Slutsky I, Farrow K, Strooper BD, Wit J. Secreted amyloid-beta precursor protein functions as a GABABR1a ligand to modulate synaptic transmission. Science, 363, eaao4827 (2019).

14) Dempsey C, Rubio Araiz A, Bryson KJ, Finucane O, Larkin C, Mills EL, Robertson AAB, Cooper MA, O’Neill LAJ, Lynch MA. Inhibiting the NLRP3 inflammasome with MCC950 promotes non-phlogistic clearance of amyloid- $\beta$ and cognitive function in APP/PS1 mice. Brain Behav. Immun., 61, 306-316 (2017).

15) National Pharmacopoeia Committee. Pharmacopoeia of the People's Republic of China [M]. Part 1. China Medical Science and Technology Press, Beijing, pp. 315-316 (2015).

16) Yao YJ, Wang YM, Kong L, Chen YQ, Yang JX. Osthole decreases tau protein phosphorylation via PI3K/AKT/GSK-3 $\beta$ signaling pathway in Alzheimer's disease. Life Sci., 217, 16-24 (2019).

17) Xie L, Sun JB, Zeng P, Liu J, Chen ZH, Zhou J. Determination of osthole in mouse brain tissue using HPLC using cloud point extrac- 
tion with optimization by response surface methodology. Pharmaceutical Journal of Chinese People's Liberation Army, 28, 205-210 (2012).

18) Jarząb A, Grabarska A, Skalicka-Woźniak K, Stepulak A. Pharmacological features of osthole. Postepy Hig. Med. Dosw., 71, 411-421 (2017).

19) Lin LW, Kuo YH, Hseu YC, Tsai CW, Hsieh MT, Chen SC, Wu CR. Osthole improves spatial memory deficits in rats via hippocampal $\alpha_{1}$-adrenergic and $\mathrm{D}_{1} / \mathrm{D}_{2}$ receptors. Evid. Based Complement. Alternat. Med., 2013, 273682 (2013).

20) Guan JH, Wei XT, Qu ST, Lv T, Fu Q, Yuan Y. Osthole prevents cerebral ischemia/reperfusion injury via Notch signaling pathway. Biochem. Cell Biol., 95, 459-467 (2017).

21) Liu H, Xue XH, Shi HJ, Qi LF, Gong DR. Osthole upregulates BDNF to enhance adult hippocampal neurogenesis in APP/PS1 transgenic mice. Biol. Pharm. Bull., 38, 1439-1449 (2015).

22) Cai M, Lee JH, Yang EJ. Bee venom ameliorates cognitive dysfunction caused by neuroinflammation in an animal model of vascular dementia. Mol. Neurobiol., 54, 5952-5960 (2017).

23) Wang X, Zhou X, Li GY, Zhang Y, Wu YL, Song WH. Modifications and trafficking of APP in the pathogenesis of Alzheimer's disease. Front. Mol. Neurosci., 10, 294 (2017).

24) Yan LL, Deng YY, Gao JM, Liu YG, Li F, Shi JS, Gong QH. Icariside II effectively reduces spatial learning and memory impairments in Alzheimer's disease model mice targeting beta-amyloid production. Front. Pharmacol., 8, 106 (2017).
25) Ali MY, Jannat S, Edraki N, Das S, Chang WK, Kim HC, Park SK, Chang MS. Flavanone glycosides inhibit $\beta$-site amyloid precursor protein cleaving enzyme 1 and cholinesterase and reduce $\mathrm{A} \beta$ aggregation in the amyloidogenic pathway. Chem. Biol. Interact., $\mathbf{3 0 9}$, 108707 (2019).

26) Nigam SM, Xu SH, Kritikou JS, Marosi K, Brodin L, Mattson MP. Exercise and BDNF reduce $\mathrm{A} \beta$ production by enhancing $\alpha$-secretase processing of APP. J. Neurochem., 142, 286-296 (2017).

27) Goldblatt G, Cilenti L, Matos JO, Lee B, Ciaffone N, Wang QX, Tetard L, Teter K, Tatulian SA. Unmodified and pyroglutamylated amyloid $\beta$ peptides form hypertoxic hetero-oligomers of unique secondary structure. FEBS J., 284, 1355-1369 (2017).

28) Taniguchi K, Yamamoto F, Arai T, Yang JW, Sakai Y, Itoh M, Mamada N, Sekiguchi M, Yamada D, Saitoh A, Kametani F, Tamaoka A, Araki YM, Wada K, Mizusawa H, Araki W. Tyrosol reduces amyloid- $\beta$ oligomer neurotoxicity and alleviates synaptic, oxidative, and cognitive disturbances in Alzheimer's disease model mice. $J$. Alzheimers Dis., 70, 937-952 (2019).

29) Zuroff L, Daley D, Black KL, Koronyo-Hamaoui M. Clearance of cerebral $\mathrm{A} \beta$ in Alzheimer's disease: reassessing the role of microglia and monocytes. Cell. Mol. Life Sci., 74, 2167-2201 (2017).

30) Aftabizadeh M, Tatarek-Nossol M, Andreetto E, El Bounkari O, Kipp M, Beyer C, Latz E, Bernhagen J, Kapurniotu A. Blocking inflammasome activation caused by $\beta$-amyloid peptide $(\mathrm{A} \beta)$ and islet amyloid polypeptide (IAPP) through an IAPP mimic. ACS Chem. Neurosci., 10, 3703-3717 (2019). 